\title{
Variabilidade espacial de atributos físico-químicos de um Latossolo Vermelho-Amarelo distrófico em sistema plantio direto
}

\section{Spatial variability of chemical and physical attributes of dystrophic Red-Yellow Latosol in no tillage}

\author{
João Vidal de Negreiros Neto ${ }^{1 *}$; Antonio Clementino dos Santos²; \\ Analu Guarnieri ${ }^{3}$; Djalma Júnior de Almeida Tavares Souza ${ }^{3}$; \\ Douglas José Daronch ${ }^{1}$; Michel Antônio Dotto ${ }^{1}$; Aridouglas dos Santos Araújo ${ }^{4 *}$
}

\section{Resumo}

O conhecimento da variabilidade espacial dos atributos químicos e físicos no solo é muito importante, principalmente, para a agricultura de precisão. A geoestatística vem em busca de aprimorar técnicas que possam viabilizar o uso correto e responsável do solo. Assim, durante o ano agrícola 2011/2012, em área de plantio de direto com a cultura do milho, no município de Gurupi (TO), no Cerrado brasileiro, objetivou-se analisar a variabilidade espacial de atributos físico-químicos de um Latossolo VermelhoAmarelo distrófico sob plantio direto. Foi instalada a malha de amostragem para a coleta de dados do solo, com 100 pontos amostrais, numa área de $1755 \mathrm{~m}^{2}$. Foram determinados os teores de $\mathrm{P}$ disponível,

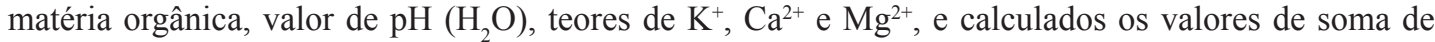
bases (SB) e saturação por bases (V\%), na profundidade de $0-0,20 \mathrm{~m}$, resistência do solo à penetração (RP), nas profundidades de $0-0,05 \mathrm{~m}, 0,05-0,10 \mathrm{~m}, 0,10-0,20 \mathrm{~m}$ e $0,20-0,40 \mathrm{~m}$ e densidade do solo na profundidade de $0-0,10 \mathrm{~m}$ (Ds). Efetuou-se a análise descritiva dos dados e em seguida foram modelados semivariogramas para todos os atributos, obtendo-se as respectivas validações cruzadas e mapas de krigagens. Os atributos químicos e físicos do solo, exceto a saturação por bases (V\%), apresentaram dependência espacial. Provavelmente, a descontinuidade da dependência espacial para $V \%$, seja devido a fertilização ao longo dos anos.

Palavras-chave: Geoestatística, densidade do solo, manejo da fertilidade do solo, Zea Mays

\begin{abstract}
Knowledge of spatial variability in chemical and physical properties of the soil is very important, especially for precision agriculture. Geostatistics is seeking to improve techniques that can enable the correct and responsible use of soil. So during the agricultural year 2011/2012 in an area of direct planting the corn crop in the municipality of Gurupi (TO), in the Brazilian Cerrado, aimed to analyze the spatial variability of chemical and physical properties in a Typic Dystrophic tillage. Was installed sampling grid for the collection of soil, with 100 sampling points in an area of $1755 \mathrm{~m}^{2}$. The contents of available phosphorus, organic matter, $\mathrm{pH}(\mathrm{H} 2 \mathrm{O})$, concentrations of $\mathrm{K}^{+}, \mathrm{Ca}^{2+}, \mathrm{Mg}^{2+}$, the sum of values and base saturation (BS, V) at depths of $0-0.20 \mathrm{~m}$, and resistance to penetration (RP) at depths $0-0.05 \mathrm{~m}$, $0.05-0.10 \mathrm{~m}, 0.10-0.20 \mathrm{~m}$ and $0.20-0.40 \mathrm{~m}$ and bulk density (Ds). We conducted a descriptive analysis

\footnotetext{
${ }^{1}$ Discentes do Curso de Doutorado em Produção Vegetal, Universidade Federal do Tocantins, UFT, Gurupi, TO. E-mail: joao_ vidal@uol.com.br; douglasdaronch@hotmail.com; micheldotto@hotmail.com; aridouglas@uft.edu.br

2 Prof. da UFT, Araguaína, TO. E-mail: clementino@uft.edu.br

3 Discentes do Curso de Mestrado em Produção Vegetal, UFT, Gurupi, TO. E-mail: analuguarnieri@gmail.com; djalmajunior@uft. edu.br

${ }^{4}$ Discente do Curso de Doutorado em Produção Animal, UFT, Araguaína, TO. E-mail: aridouglas@uft.edu.br

* Autor para correspondência
} 
classic, with the aid of statistical software ASSISTAT, and then were modeled semivariograms for all attributes, resulting in their cross-validation and kriging maps. The chemical and physical properties of soil, except the base saturation (V), spatial dependence. Probably the discontinuity of the spatial dependence of Vvalue, is due to fertility management over the years.

Key words: Geostatistics, bulk density, soil fertility management, Zea Mays

\section{Introdução}

A utilização do solo no sistema convencional de cultivo altera seus atributos químicos originais, pelo manejo de corretivos e fertilizantes (COUTO, 1997; ARAÚJO et al., 2011). Nos últimos anos, os métodos de preparo do solo, como o reduzido e o plantio direto, vêm sendo adotados em substituição aos convencionais. No sistema plantio direto, a variabilidade dos atributos do solo é aumentada pela ação residual das linhas de adubação, que se mantêm na sequência dos cultivos, juntamente com a redistribuição dos nutrientes reciclados dos resíduos vegetais presentes no solo (COUTO, 1997).

Os atributos do solo podem apresentar grande variabilidade espacial dependendo do solo, topografia, clima, vegetação e atividades antrópicas. O entendimento da natureza da variabilidade é indispensável para avaliar o efeito dos atributos do solo sobre a produtividade das culturas agrícolas, principalmente em regiões tropicais, onde a dinâmica dos processos e a heterogeneidade são maiores do que em outros ambientes (RICHTER; BABBAR, 1991).

Ao constatar a existência de dificuldades como numerosos atributos do solo interligados e suas interações complexas com as práticas agrícolas, Garrigues et al. (2012) observaram que tais dificuldades adiaram o estabelecimento de indicador de impacto universal da qualidade do solo na avaliação ambiental. Em ecossistemas nativos, o solo encontra-se em equilíbrio dinâmico, onde as entradas e saídas se compensam (DIAS-FILHO, 2006).

Nos estudos estatísticos iniciais da variabilidade dos atributos do solo eram utilizados modelos estatísticos simples (WEBSTER; BECKETT, 1968). Com o aprimoramento da geoestatística, a amostragem e o número adequado de amostras passou a ser fundamental para gerar modelos mais confiáveis (LIMA; SOUZA; SILVA, 2010b). Estes autores constataram que o número de amostras na estatística clássica foi maior se comparado com a geoestatística. Corroborando com essa vertente, Leão et al. (2007) melhoraram a eficácia das estratégias de amostragem com a incorporação de um modelo de variabilidade espacial dos atributos do solo, possibilitando a aplicação desse conhecimento na escolha da área experimental, locação de unidades experimentais, coleta de amostras e interpretação de resultados. Além disso, o conhecimento da variabilidade espacial dos atributos da fertilidade do solo pode contribuir na definição de melhores estratégias para o manejo sustentável do solo (SCHAFFRATH et al., 2008).

Com base no exposto, o objetivo deste trabalho foi avaliar a variabilidade espacial dos atributos químicos e físicos do solo em área cultivada com milho no sistema plantio direto, por meio da estatística clássica e da geoestatística.

\section{Material e Métodos}

O experimento foi instalado na área experimental da Universidade Federal do Tocantins (UFT), Campus Universitário de Gurupi, localizado na região Sul do estado do Tocantins, a $280 \mathrm{~m}$ de altitude, abrangendo as coordenadas geográficas $11^{\circ} 43^{\prime} 45^{\prime}$ ' de latitude Sul e 49 $04^{\prime} 07^{\prime \prime}$ de longitude Oeste. Segundo a classificação climática de Köppen (1948), o clima regional é do tipo b1wA'a' úmido com moderada deficiência hídrica. Conforme preceitos do Sistema Brasileiro de Classificação de Solos (EMBRAPA, 2006), o solo local é Latossolo Vermelho-Amarelo distrófico. 
O solo estudado vem sendo cultivado há oito anos com a cultura do milho, sendo que nos três primeiros anos de cultivo, o preparo do solo foi convencional, a correção do solo foi realizada buscando elevar a saturação por base a $60 \%$ e a adubação de implantação e de cobertura seguiram as exigências da cultivar plantada, e nos últimos cinco anos vêm sendo utilizado o sistema plantio direto. Foi delimitada a área experimental de 130 x 13,5 m $\left(1755 \mathrm{~m}^{2}\right)$. A malha amostral utilizada foi composta por 100 pontos, distribuídos em quatro linhas de $130 \mathrm{~m}$, com espaçamento entre pontos de coleta (na linha) de 5,2 e entre linhas de $3,37 \mathrm{~m}$.

A densidade do solo foi determinada pelo método do anel volumétrico (EMBRAPA, 1997). As amostras indeformadas foram coletadas na profundidade de $0-0,10 \mathrm{~m}$, utilizando um amostrador tipo Uhland, utilizando cilindros com $7 \mathrm{~cm}$ de diâmetro por $7 \mathrm{~cm}$ de altura e volume de $269,3 \mathrm{~cm}^{3}$. Após a coleta, as amostras foram levadas ao laboratório, colocadas em estufa a $105^{\circ} \mathrm{C}$ por 24 horas, depois de retiradas deixou-se esfriar e procedeu-se a pesagem dos cilindros. Utilizou-se a equação 1 para a determinação da densidade do solo (Ds) dada em $\mathrm{Mg} \mathrm{m}^{-3}$.

$\operatorname{Ds}\left(\mathrm{Mg} \mathrm{m}^{-3}\right)=$ massa do solo seco volume de solo

As análises dos atributos químicos do solo, $\mathrm{pH}$ (em água); $\mathrm{Ca}$ e $\mathrm{Mg}\left(\mathrm{cmol}_{\mathrm{c}} \mathrm{dm}^{-3}\right)$; fósforo disponível ( $\left.\mathrm{P} ; \mathrm{mg} \mathrm{dm}^{-3}\right)$; matéria orgânica do solo (MOS; $\left.\mathrm{g} \mathrm{kg}^{-1}\right)$; teores de potássio trocável $\left(\mathrm{K}^{+}\right.$; $\mathrm{cmol}_{\mathrm{c}} \mathrm{dm}^{-3}$ ); valores de saturação por bases ( $\mathrm{V} \%$ ); soma de bases $\left(\mathrm{SB}\right.$; $\left.\mathrm{cmol}_{\mathrm{c}} \mathrm{dm}^{-3}\right)$; capacidade de troca catiônica $\left(\mathrm{CTC}_{\mathrm{pH}}\right.$; $\left.\mathrm{cmol}_{\mathrm{c}} \mathrm{dm}^{-3}\right)$ e $\mathrm{H}+\mathrm{Al}\left(\mathrm{cmol}_{\mathrm{c}}\right.$ $\mathrm{dm}^{-3}$ ), foram feitas apartir do solo coletado no anel volumétrico e analisadas segundo o método descrito pela EMBRAPA (1997).

Para a determinação da resistência do solo à penetração (RP) foi utilizado o penetrômetro de impactos, modelo IAA/Planalsucar - Stolf. A umidade média do solo $\left(0,16 \mathrm{~kg} \mathrm{~kg}^{-1}\right)$ durante as coletas foi determinada pela diferença de massa das amostras antes e depois de serem levadas a estufa. As profundidades analisadas foram de $0-0,05 ; 0,05-$ 0,10; 0,10-0,20; 0,20-0,40 $\mathrm{m}$ de profundidade, com os valores transformados em MPa, de acordo com a metodologia proposta por Stolf, Fernandes e Furlani Neto (1983).

Inicialmente, os valores dos atributos físicoquímicos do solo foram submetidos à análise exploratória para verificar se esses ocorriam de maneira aleatória ou agregadas, calculando-se a média, mediana, assimetria, curtose e coeficiente de variação (CV). Utilizou-se o teste de KolmogorovSmirnov a 5\% de significância para testar a hipótese de normalidade. Como ferramenta estatística foi utilizado o programa Assistat 7.5 (SILVA; AZEVEDO, 2008). Foram adotados os limites do coeficiente de variação $(\mathrm{CV})$ classificados em baixo $(\mathrm{CV}<12 \%)$, médio $(12<\mathrm{CV}<60 \%)$ e alto $(\mathrm{CV}>$ $60 \%$ ), propostos por Warrick e Nielsen (1980).

A análise da dependência espacial foi feita através do ajuste dos dados ao semivariograma experimental, de acordo com a teoria das variáveis regionalizadas. Após foram feitas as interpolações dos dados por krigagem ordinária e posteriormente os gráficos de contorno, com auxílio do programa GS+ v. 5.1.1 (ROBERTSON, 1998). Utilizaram-se lags com intervalos não uniformes para os ajustes aos modelos. Realizou-se a seleção dos modelos com base na menor SQR (soma de quadrados dos resíduos) e melhor $\mathrm{R}^{2}$ (coeficiente de determinação espacial) e grau de dependência espacial:

$$
\mathrm{GDE}=\left[\mathrm{C} / \mathrm{Z}\left(\mathrm{C} \rrbracket_{0}+\mathrm{C}\right)\right] * 100
$$

onde:

GDE = grau de dependência espacial;

$\mathrm{C}_{0}=$ efeito pepita;

$\mathrm{C}=$ variância estrutural;

$\mathrm{C}_{0}+\mathrm{C}=$ patamar.

A interpretação do grau de dependência espacial para escolha do modelo foi de acordo com a 
seguinte classificação: GDE $<20 \%$, considerada dependência espacial muito baixa; $20 \% \leq \mathrm{GDE}<$ $40 \%$, considerada dependência espacial baixa; $40 \%$ $\leq$ GDE $<60 \%$, considerada dependência espacial média; $60 \% \leq \mathrm{GDE}<80 \%$, considerada dependência espacial alta; $80 \% \leq$ GDE $<100 \%$, considerada dependência espacial muito alta (DALCHIAVON e CARVALHO, 2012).

\section{Resultados e Discussão}

Os valores medianos dos atributos químicos do solo para a área em sistema plantio direto foram classificados como baixo para $\mathrm{H}+\mathrm{Al}\left(2,43 \mathrm{cmol}_{\mathrm{c}} \mathrm{dm}^{-}\right.$ $\left.{ }^{3}\right)$, médio para $\mathrm{P}$ disponível $\left(16 \mathrm{mg} \mathrm{dm}^{-3}\right), \mathrm{K}$ trocável $\left(0,15 \mathrm{cmol}_{\mathrm{c}} \mathrm{dm}^{-3}\right), \mathrm{CTC}_{\mathrm{pH} 7}\left(5,94 \mathrm{cmol}_{\mathrm{c}} \mathrm{dm}^{-3}\right)$, soma de bases $\left(3,64 \mathrm{cmol}_{\mathrm{c}} \mathrm{dm}^{-3}\right)$ e matéria orgânica do solo $(1,59 \%)$ e alto para $\mathrm{Ca}+\mathrm{Mg}\left(3,45 \mathrm{cmol}_{\mathrm{c}} \mathrm{dm}^{-}\right.$ $\left.{ }^{3}\right)$ e saturação por base $(59,93 \%)$, conforme classes de interpretação de fertilidade do solo (RIBEIRO; GUIMARÃES; ALVAREZ, 1999).

O teste de normalidade de Kolmogorov-Smirnov indicou distribuição normal dos dados apenas para as variáveis $\mathrm{CTC}_{\mathrm{pH} 7}$ e densidade do solo (Tabela 1). Estudando a espacialidade de dados de fertilidade do solo no Norte do Tocantins, Silva-Neto et al. (2011) evidenciaram que somente a $\mathrm{CTC}_{\mathrm{pH} 7}$, CTC efetiva e matéria orgânica do solo apresentaram distribuição normal (Kolmogorov-Smirnov), assim como MeloFilho et al. (2006) verificaram distribuição normal (Kolmogorov-Smirnov) para os dados de densidade do solo em estudo realizado em Cruz das Almas, BA.

A ausência de normalidade nos atributos químicos do solo deve-se, provavelmente, ao histórico de adubação desuniforme da área em estudo, por se tratar de área experimental, onde no passado houve diferentes manejos do solo. No entanto, de acordo com Corá e Beraldo (2006), não é necessária a distribuição normal das variáveis no estudo geoestatístico, quando se avalia os dados em conjunto.

Os atributos $\mathrm{Ca}+\mathrm{Mg}, \mathrm{H}+\mathrm{Al}, \mathrm{CTC}_{\mathrm{pH} 7}$, soma de bases e densidade do solo apresentaram coeficiente de assimetria negativa (Tabela 1), o que demonstra tendência de considerável quantidade de valores maiores que a média amostral. Os demais atributos químicos e físicos do solo apresentaram distribuição assimétrica positiva.

Os coeficientes de curtose são utilizados para avaliar se os dados seguem distribuição normal (ORTIZ, 2003). Os valores de curtose devem, preferencialmente, ser nulos, podendo ser aceitos valores entre +2 e -2 . Observa-se na Tabela 1 que os valores de coeficientes de curtose de $\mathrm{Ca}+\mathrm{Mg}$, $\mathrm{K}^{+}$, soma de bases e de matéria orgânica do solo, bem como, para a resistência do solo à penetração em todas as profundidades não se apresentaram nos limites indicados por Ortiz (2003).

A variabilidade dos dados pode ser medida pela variância e pelo coeficiente de variação, considerados os primeiros indicativos de heterogeneidade dos dados (BERNER et al., 2007). O coeficiente de variação da densidade do solo (7,93\%) é considerado baixo (WARRICK; NIELSEN, 1980). Esse coeficiente é importante na definição de quantidades de amostras que devem ser coletadas, obtendo-se, dessa forma, um valor médio representativo.

Observa-se que os valores da média e mediana de todas as variáveis são próximos entre si, caracterizando distribuição tendendo a simetria, concordando com Campos et al. (2010), que observaram, para as variáveis $\mathrm{pH}$, saturação por base e matéria orgânica do solo, simetria na distribuição dos dados.

Desta forma, torna-se indispensável, nesse estudo, a análise do efeito proporcional, quando a média e a variabilidade dos dados são constantes na área em estudo (SILVA et al., 2003). Sob este aspecto, observa-se na Tabela 2 que os semivariogramas resultantes dos dados apresentaram patamares bem definidos e a distribuição não apresentou caudas muito alongadas, contribuindo para estimativas de krigagem ordinária, que levam em conta os valores médios (ISAAKS; SRIVASTAVA, 1989). 
Tabela 1. Análise descritiva dos atributos químicos e físicos do Latossolo Vermelho Amarelo distrófico.

\begin{tabular}{lccccccr}
\hline \multirow{2}{*}{ Variável } & \multirow{2}{*}{ Média } & \multirow{2}{*}{ Mediana } & \multirow{2}{*}{ Variância } & \multicolumn{3}{c}{ Coeficiente } & \multirow{2}{*}{ KS $^{\mathbf{1}}$} \\
\cline { 5 - 6 } & & & & Variação & Assimetria & Curtose & \\
\hline $\mathrm{Ca}+\mathrm{Mg}$ & 3,35 & 3,45 & 0,73 & 21,80 & $-1,24$ & 2,69 & $0,01^{\text {ns }}$ \\
$\mathrm{pH}\left(\mathrm{H}_{2} \mathrm{O}\right)$ & 5,89 & 5,86 & 0,25 & 8,54 & 0,51 & $-0,54$ & $0,05^{\text {ns }}$ \\
$\mathrm{H}+\mathrm{Al}$ & 2,29 & 2,43 & 0,62 & 34,38 & $-0,51$ & $-0,20$ & $0,01^{\text {ns }}$ \\
$\mathrm{P}$ & 15,66 & 16,00 & 28,98 & 34,37 & 0,21 & 1,44 & $0,01^{\text {ns }}$ \\
$\mathrm{K}$ & 0,15 & 0,15 & 0,006 & 50,53 & 1,45 & 3,41 & $0,01^{\text {ns }}$ \\
$\mathrm{CTC}$ & 5,79 & 5,94 & 1,27 & 19,47 & $-0,71$ & 1,13 & $0,05^{*}$ \\
$\mathrm{SB}$ & 3,50 & 3,64 & 0,55 & 21,22 & $-1,27$ & 2,68 & $0,025^{\text {ns }}$ \\
V & 60,60 & 59,93 & 135,83 & 19,23 & 0,07 & 1,46 & $0,05^{\text {ns }}$ \\
MOS & 2,00 & 1,59 & 1,12 & 52,87 & 2,13 & 5,98 & $0,01^{\text {ns }}$ \\
Ds & 2,46 & 2,47 & 0,04 & 7,93 & $-0,03$ & 0,15 & $0,15^{*}$ \\
RP 0,05 m & 0,72 & 0,61 & 0,17 & 57,00 & 1,68 & 2,78 & $0,01^{\text {ns }}$ \\
RP 0,10 m & 1,39 & 1,23 & 0,48 & 49,87 & 2,15 & 6,32 & $0,01^{\text {ns }}$ \\
RP 0,20 m & 2,57 & 2,24 & 2,09 & 56,29 & 5,21 & 39,28 & $0,01^{\text {ns }}$ \\
RP 0,40 m & 3,29 & 2,80 & 3,50 & 56,81 & 4,03 & 20,56 & $0,01^{\text {ns }}$ \\
\hline
\end{tabular}

${ }^{(1)} \mathrm{KS}$ : teste de normalidade de Kolmogorov-Smirnov; (*): significativo a 5\%: ( ${ }^{\mathrm{ns}}$ ): não-significativo; $\mathrm{Ca}+\mathrm{Mg}, \mathrm{K}, \mathrm{H}+\mathrm{Al}, \mathrm{CTC} \mathrm{pH}_{7}$ (capacidade de troca de cátions a $\mathrm{pH}$ 7), $\mathrm{SB}$ (soma de bases) em $\mathrm{cmol}_{\mathrm{c}} \mathrm{dm}^{-3}$ : P disponível em $\mathrm{mg} \mathrm{dm}^{-3}$ : V\% (saturação por bases) e MOS (matéria orgânica do solo) em \%: Ds (densidade do solo) em $\mathrm{Mg} \mathrm{m}^{-3}$ : e RP (resistência do solo à penetração) em MPa.

Fonte: Elaboração dos autores.

Para os atributos químicos do solo que apresentaram grau de dependência espacial alto $\left(\mathrm{Ca}+\mathrm{Mg}, \mathrm{CTC}_{\mathrm{pH}}\right.$, densidade do solo e resistência do solo à penetração, nas profundidades de 0-0,05 e 0,05-0,10 m), foram ajustadoso modelo semivariográfico exponencial (Tabela 2), exceto para a $\mathrm{CTC}_{\mathrm{pH} 7}$ (esférico), para as demais variáveis o grau de dependência espacial foi médiana. A saturação por bases apresentou efeito pepita puro, indicando que a distância de amostragem não foi suficiente para exibir toda a variância dos dados. Esta descontinuidade na distribuição da dependência espacial, provavelmente esteja relacionada ao manejo da fertilidade do solo ao longo dos anos, cuja adubação seguiu as linhas de plantio e a exigência da cultivar plantada.

Os valores do grau de dependência espacial revelam que as propriedades intrínsecas do solo, como material de origem, clima, relevo e organismos, diminuíram de importância, indicando maior relevância das suas propriedades extrínsecas dos efeitos dos manejos adotados ao longo do tempo de uso do solo (CAMBARDELLA et al., 1994).

Para os dados de resistência do solo à penetração, em todas as profundidades, foi ajustado o modelo esférico, e para os dados da densidade do solo, modelo exponencial (Tabela 2). Assim, os atributos proporcionaram ajustes semivariográficos com dependência espacial média, em sua maioria (DALCHIAVON; CARVALHO, 2012). Tais ajustes corroboram, em parte, com os apresentados por Souza, Marques-Júnior e Pereira (2004), Souza et al. (2006) e Cruz et al. (2010), que verificaram predominância de modelos exponencial e gaussiano.

O alcance variou de 19,7 (P) a 30,6 m $\left(\mathrm{CTC}_{\mathrm{pH}}\right)$, conforme verifica-se na Tabela 2. O alcance é um indicador do limite da dependência que a variável apresenta no espaço. Significa que determinações realizadas a distâncias maiores que o alcance, possui distribuição espacial aleatória, dessa forma, são independentes entre si; por outro lado, determinações realizadas em distâncias menores que o alcance, são correlacionadas umas às outras, o que permite que se façam interpolações para espaçamentos menores que os amostrados (CAMPOS et al., 2008).

Valores de alcance muito baixos podem acarretar queda na qualidade das estimativas, visto que poucos pontos são usados na interpolação para estimar os valores em locais não medidos (CORÁ et al., 2004). No presente estudo, os valores do alcance 
dos atributos dos solos apresentaram semelhanças nos limites da área estudada.

Os mapas temáticos de isolinhas obtidos por meio da interpolação por krigagem para os atributos químicos e físicos do solo são apresentados nas Figuras 1 e 2, respectivamente. Na Figura 1, os mapas de isolinhas permitiram a visualização de zonas com os maiores e menores valores para os atributos. Maiores teores de $\mathrm{Ca}+\mathrm{Mg}, \mathrm{K}, \mathrm{CTC}_{\mathrm{pH} 7}$ e $\mathrm{SB}$ foram observados próximo ao canto esquerdo superior, coincidindo com área próxima à leira formada durante a construção de um terraço de contenção da erosão.

Tabela 2. Modelos e parâmetros estimados dos semivariogramas ajustados aos valores dos atributos químicos e físicos do solo.

\begin{tabular}{|c|c|c|c|c|c|c|c|c|}
\hline \multirow[b]{2}{*}{ Atributo } & \multicolumn{8}{|c|}{ Parâmetro } \\
\hline & Modelo & $\mathrm{C}_{0}{ }^{1}$ & $\mathrm{C}_{0}+\mathrm{C}^{2}$ & $\begin{array}{c}\text { GDE }^{3} \\
(\%)\end{array}$ & Classe & $\mathbf{A}^{4}(\mathbf{m})$ & $\mathbf{R}^{2 *}$ & $\mathrm{SQR}^{5}$ \\
\hline $\mathrm{Ca}+\mathrm{Mg}$ & Exponencial & 0,0905 & 0,342 & 73,54 & Alta & 21,2 & 0,627 & $2,07^{-2}$ \\
\hline $\mathrm{pH}$ & Exponencial & 0,1127 & 0,260 & 57,31 & Média & 20,5 & 0,576 & $1,54^{-2}$ \\
\hline $\mathrm{H}+\mathrm{Al}$ & Exponencial & 0,329 & 0,700 & 53,00 & Média & 21,9 & 0,463 & $5,36^{-2}$ \\
\hline $\mathrm{P}$ & Exponencial & 13,85 & 31,800 & 56,45 & Média & 19,7 & 0,444 & 144 \\
\hline $\mathrm{K}^{+}$ & Exponencial & 0,00296 & 0,0063 & 53,02 & Média & 20,6 & 0,538 & $9,73^{-6}$ \\
\hline $\mathrm{CTC}_{\mathrm{pH} 7}$ & Esférico & 0,416 & 1,330 & 68,72 & Alta & 30,6 & 0,521 & 0,35 \\
\hline $\mathrm{SB}^{\mathrm{pH} 7}$ & Exponencial & 0,256 & 0,600 & 57,33 & Média & 22,0 & 0,428 & $4,89^{-2}$ \\
\hline $\mathrm{V} \%$ & Efeito pepita puro & - & - & - & - & - & - & - \\
\hline MOS & Exponencial & 0,528 & 1,207 & 56,25 & Média & 21,3 & 0,547 & 0,65 \\
\hline Ds & Exponencial & 0,0124 & 0,044 & 71,82 & Alta & 21,5 & 0,490 & $3,51^{-4}$ \\
\hline RP 5 & Esférico & 0,062 & 0,170 & 63,53 & Alta & 20,9 & 0,782 & $7,29^{-3}$ \\
\hline RP 10 & Esférico & 0,181 & 0,485 & 62,68 & Alta & 22,2 & 0,731 & 0,12 \\
\hline RP 20 & Esférico & 0,847 & 2,100 & 59,67 & Média & 20,4 & 0,520 & 1,19 \\
\hline RP 40 & Esférico & 1,91 & 3,261 & 41,42 & Média & 21,6 & 0,725 & 21,9 \\
\hline
\end{tabular}

${ }^{(1)} \mathrm{C}_{0}$ : efeito pepita; ${ }^{(2)} \mathrm{C}_{0}+\mathrm{C}$ : patamar; ${ }^{(3)} \mathrm{GDE}$ : grau de dependência espacial; ${ }^{(4)} \mathrm{A}$ : alcance; ${ }^{(5)} \mathrm{SQR}$ : soma dos quadrados dos resíduos; (*) $\mathrm{R}^{2}$ : coeficiente de determinação espacial; $\mathrm{Ca}+\mathrm{Mg}, \mathrm{K}, \mathrm{H}+\mathrm{Al}, \mathrm{CTC}_{\mathrm{pH}}$ (capacidade de troca de cátions a pH 7), $\mathrm{SB}$ (soma de bases); P disponível; V\% (saturação por bases) e MOS (matéria orgânica do solo); Ds (densidade do solo); e RP (resistência do solo à penetração).

Fonte: Elaboração dos autores.

Em algumas regiões do mapa o teor de matéria orgânica do solo observado foi baixo (0,40 a 1,6\%), representando a maior área, em locais isolados foi observado teor de matéria orgânica de 5,2 a 6,4\% (Figura 1). As baixas concentrações de matéria orgânica estão relacionadas ao tempo de implantação do sistema plantio direto (cinco anos), ainda não consolidado, por causa das condições ambientais nos trópicos que favorecem a rápida decomposição do material orgânico adicionado ao solo, dificultando o acúmulo de matéria orgânica, por mais que sejam adotados sistemas que a preservam (MOURA et al., 2010; GUARESCHI; PEREIRA; PERIN, 2012).
Em ambientes tropicais, estima-se de 9 a 10 anos de sua implantação para consolidação de sistemas de plantio direto (PEREIRA NETO et al., 2007).

A densidade do solo apresentou valor elevado $\left(2,47 \mathrm{Mg} \mathrm{m}^{-3}\right)$ se comparada com outros trabalhos em condições de plantio direto em cerrado (RALISCH et al., 2008; TORMENA; BARBOSA; COSTA, 2002; WEIRICH-NETO; ROSA; GOMES, 2002), conforme a Tabela 1. As operações com maquinários e implementos na área podem ter ocasionado elevação nos valores de densidade, que em praticamente toda área do mapa estiveram entre 2,2 e 2,5 $\mathrm{Mg} \mathrm{m}^{-3}$ (Figura 2). 
Figura 1. Mapas de contorno da distribuição espacial de atributos químicos de um Latossolo Vermelho-Amarelo distrófico.
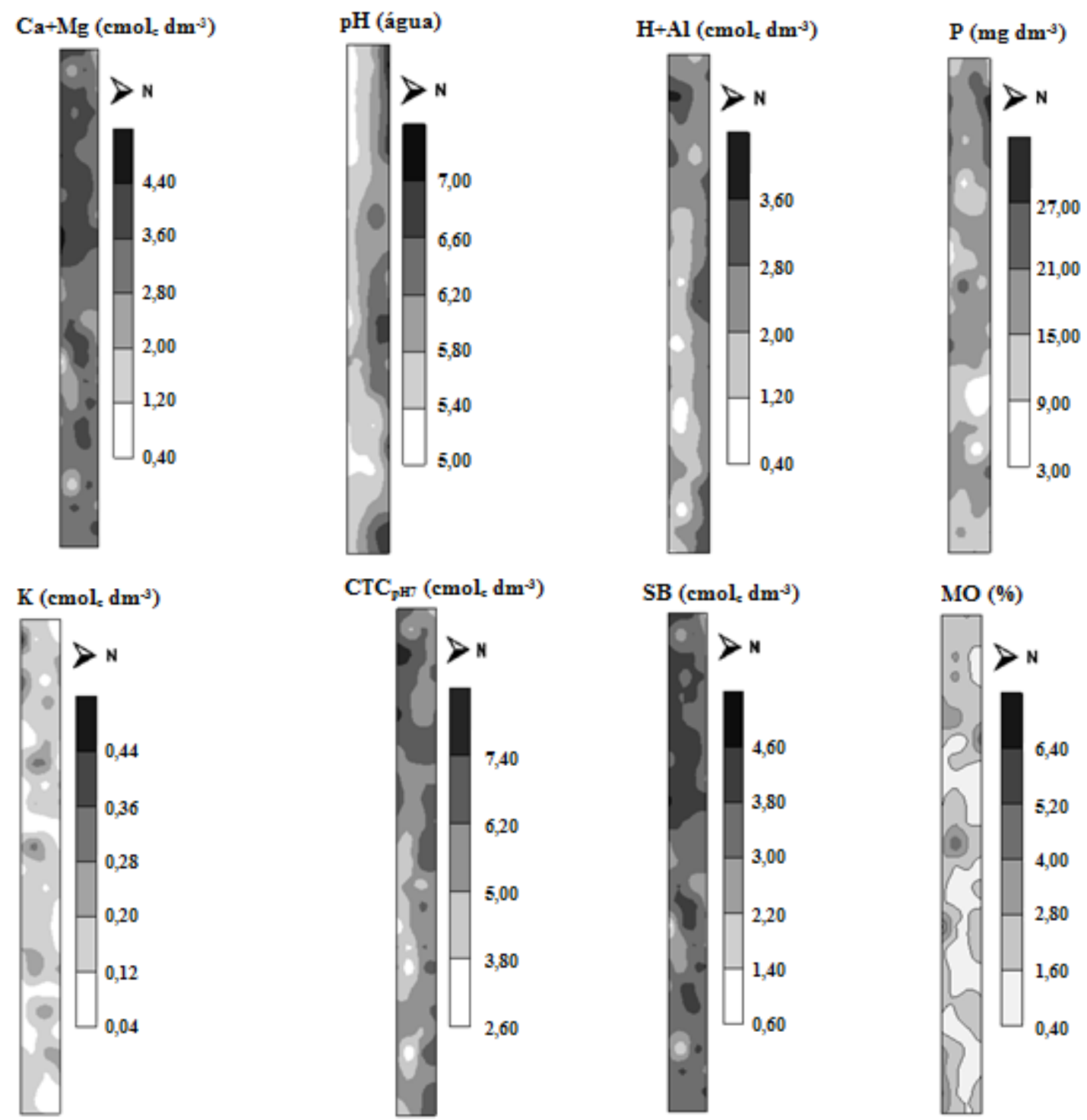

Fonte: Elaboração dos autores. 
Figura 2. Mapas de contorno da distribuição espacial dos atributos físicos de um Latossolo Vermelho-Amarelo distrófico.
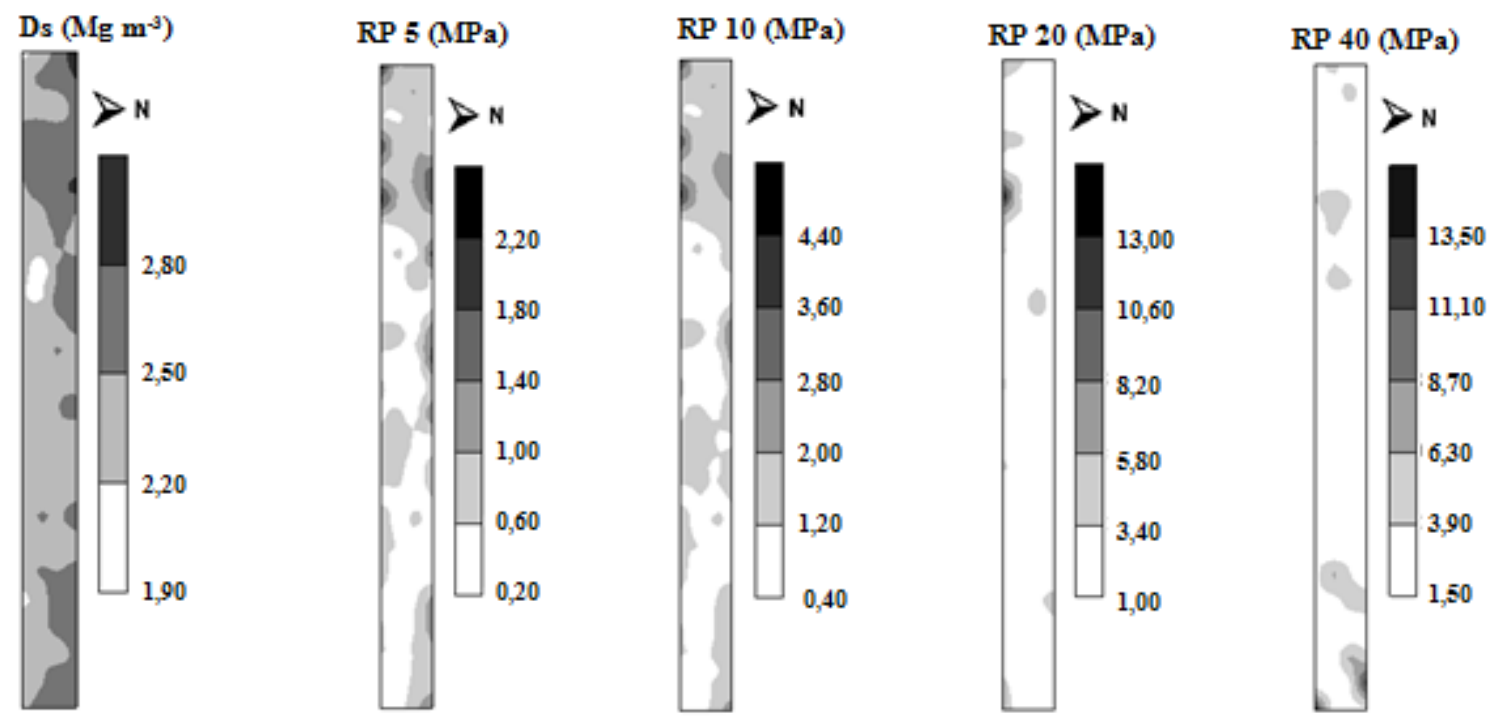

Fonte: Elaboração dos autores.

As raízes do milho que se desenvolvem em um solo com densidade maior que $1,2 \mathrm{Mg} \mathrm{m}^{-3}$ não terão um grau elevado de ramificação de raízes ou formação de raízes secundárias, o que não permitiriam que as raízes explorassem o solo, prejudicando a absorção, principalmente àqueles de pouca mobilidade como o fósforo (RICHART et al., 2005). Esse processo diminui a produtividade do milho devido à deficiência de nutrientes na planta, provocado pelo impedimento do crescimento radicular e menor exploração do solo.

Os valores de resistência do solo à penetração foram baixos na camada de 0-0,05 m e compatíveis com os apresentados por Ralisch et al. (2008), com valores menores em superfície, mas com acréscimos em profundidade, onde, na camada de 0,05-0,10, 0,10-0,20 e 0,20-0,40 m seus valores, 1,23, 2,24 e 2,80 $\mathrm{MPa}$, respectivamente, foram críticos, considerando que valores acima de 1,70 MPa são limitantes à produção de espécies cultivadas (LIMA et al., 2010a). Tormena, Barbosa e Costa (2002) apresentaram valores de resistência do solo à penetração com umidade de $0,16 \mathrm{~kg} \mathrm{~kg}^{-1}$ teve comportamentos diferentes aos da presente pesquisa, em sistema plantio direto, com tendência de redução de valores em profundidade. A diferença encontrada entre os autores se deve ao implemento utilizado no revolvimento do solo no preparo inicial e intensidade do tráfego de máquinas ao longo do tempo (TORMENA; ROLOFF; SÁ, 1998).

$\mathrm{Na}$ Figura 2 é possível identificar regiões de densidade do solo mais elevadas, coincidentes com os mapas de resistência do solo à penetração, em todas as profundidades estudadas (RP 5, RP 10, RP 20 e RP 40). Esses resultados são semelhantes àqueles apresentados por Tormena, Barbosa e Costa (2002), que relacionaram os atributos físicos densidade do solo e resistência do solo à penetração.

$\mathrm{Na}$ área superior direita, no mapa de $\mathrm{P}$ disponível, ocorreram teores mais elevados. Essa região coincide com o ponto mais baixo da área estudada, indicando que houve maior concentração de $\mathrm{P}$ disponível. Apesar da pouca mobilidade do $\mathrm{P}$ no solo, o escoamento superficial da água pode ter carreado solo e nutrientes para áreas de acúmulo, podendo ser uma das causas da heterogeneidade do nutriente na área. 
A heterogeneidade da distribuição dos nutrientes e dos atributos físicos do solo verificados pode afetar negativamente a produção de grãos, tornando-a desuniforme dentro da área cultivada. Porém com a verificação dos locais de maior e menor concentração de nutrientes poderá ser realizada o manejo da adubação buscando-se uniformizar os teores dos nutrientes na área, além de intervenções para diminuir a densidade do solo e a resistência do solo à penetração para valores que permitam o adequado desenvolvimento radicular do milho.

\section{Conclusões}

Excetuando-se a saturação por bases, todos os atributos químicos e físicos do solo apresentaram dependência espacial, indicando a importância das distâncias entre as amostras para efeito de fertilidade e física do solo;

A descontinuidade na distribuição espacial da saturação por bases, provavelmente está relacionada ao manejo da fertilização ao longo dos anos;

A análise criteriosa dos atributos físico-químicos do solo, por meio dos indicadores da distribuição espacial, permitiu identificar os locais de deficiência de nutrientes e os impedimentos físicos provocados pela elevada densidade do solo e resistência do solo à penetração. E não houve contribuição do sistema de plantio direto após cinco anos da implantação.

\section{Agradecimentos}

Ao $\mathrm{CNPq}$ e a CAPES pelo apoio e bolsas concedidas.

\section{Referências}

ARAÚJO, A. S.; SILVA, J. E. C.; SANTOS, A. C.; SILVA-NETO, S. P.; DIM, V. P.; ALEXANDRINO, E. Substituição de nitrogênio por esterco bovino na produtividade de forragem e qualidade do solo. Revista Brasileira de Saúde e Produção Animal, Salvador, v. 12, n. 4, p. 852-66, 2011.
BERNER, P. G. M.; VIEIRA, S. R.; LIMA, E.; ANJOS, L. H. C. Variabilidade espacial de propriedades físicas e químicas de um Cambissolo sob dois sistemas de manejo de cana-de-açúcar. Revista Brasileira de Ciência do Solo, Viçosa, v. 31, n. 5, p. 415-422, 2007.

CAMBARDELLA, C. A.; MOORMAN, T. B.; NOVAK, J. M.; PARKIN, T. B.; KARLEN, D. L.; TURCO, R. F.; KONOPKA, A. E. Field-scale variability of soil properties in Central Iowa Soils. Soil Science Society of American Journal, Madison, v. 58, n. 5, p. 1501-1511, 1994.

CAMPOS, M. C. C.; MARQUES-JÚNIOR, J.; PEREIRA, G. T.; SOUZA, Z. M.; BARBIERI, D. M. Aplicação de adubo e corretivo após o corte da canaplanta utilizando técnicas geoestatísticas. Ciência Rural, Santa Maria, v. 38, n. 4, p. 974-980, 2008.

CAMPOS, S.; NARDINI, R. C.; BARROS, Z. X.; CARDOSO, L. G. Sistema de informações geográficas aplicado à especialização da capacidade de uso da terra. Pesquisa Agropecuária Tropical, Goiânia, v. 40, n. 2, p. 174-179, 2010.

CORÁ, J. E.; ARAUJO, A. V.; PEREIRA, G. T.; BERALDO, J. M. G. Variabilidade espacial de atributos do solo para adoção do sistema de agricultura de precisão na cultura de cana-de-açúcar. Revista Brasileira de Ciência do Solo, Viçosa, v. 28, n. 6, p. 1013-1021, 2004.

CORÁ, J. E.; BERALDO, J. M. Variabilidade espacial de atributos do solo antes e após calagem e fosfatagem em doses variadas na cultura de cana-de-açúcar. Engenharia Agrícola, Jaboticabal, v. 26, n. 2, p. 374-387, 2006.

COUTO, E. G. Variabilidade espacial de propriedades do solo influenciadas pela agricultura em escala regional e local no sul do estado do Mato Grosso. 1997. Tese (Doutorado em Ciência do Solo) - Universidade Federal do Rio Grande do Sul, Porto Alegre.

CRUZ, J. S.; ASSIS-JÚNIOR, R. N.; MATIAS, S. S. R.; TAMAYO, J. H. C.; TAVARES, R. C. Análise espacial de atributos físicos e carbono orgânico em Argissolo Vermelho-Amarelo cultivado com cana-de-açúcar. Ciência e Agrotecnologia, Lavras, v. 34, n. 2, p. 361-368, 2010.

DALCHIAVON, F. C.; CARVALHO, M. P. Correlação linear e espacial dos componentes de produção e produtividade da soja. Semina: Ciências Agrárias, Londrina, v. 33, n. 2, p. 541-552, 2012.

DIAS-FILHO, M. B. Sistemas silvipastoris na recuperação de pastagens degradadas. Embrapa Amazônia Oriental, 2006. 31 p. 
EMPRESA BRASILEIRA DE PESQUISA AGROPECUÁRIA - EMBRAPA. Manual de métodos de análise de solo. Centro Nacional de Pesquisa de Solo. 2. ed. Rio de Janeiro: Embrapa CNPS, 1997. 212 p.

Sistema brasileiro de classificação de solos. 2 . ed. Brasília: Embrapa Produção de Informação. Rio de Janeiro: Embrapa Solos, 2006. 306 p.

GARRIGUES, E.; CORSON, M. S.; ANGERS, D. A.; VAN DER WERF, H. M. G.; WALTER, C. Soil quality in Life Cycle Assessment: Towards development of an indicator. Ecological Indicators, v. 18, n. 7, p. 434-442, 2012.

GUARESCHI, R. F.; PEREIRA, M. G.; PERIN, A. Deposição de resíduos vegetais, matéria orgânica leve, estoque de carbono e nitrogênio e fósforo remanescente sob diferentes sistemas de manejo no cerrado goiano. Revista Brasileira de Ciência do solo, Viçosa, v. 36, n. 3, p. 909-920, 2012.

ISAAKS, E. H.; SRIVASTAVA, R. M. An introduction to applied geoestatistics. New York: Oxford University, 1989. $561 \mathrm{p}$.

LEÃO, A. B.; ANDRADE, A. R. S.; CHAVES, L. H. G.; CHAVES, I. B.; GUERRA, H. O. C. Variabilidade espacial de fósforo em solo do perímetro irrigado Engenheiro Arcoverde, PB. Revista Ciência Agronômica, Fortaleza, v. 38, n. 1, p. 1-6, 2007.

LIMA, C. L. R.; REINERT, D. J.; REICHERT, J. M.; SUZUKI, L. E. A. S. Produtividade de culturas e resistência à penetração de Argissolo Vermelho sob diferentes manejos. Pesquisa Agropecuária Brasileira, Brasília, v. 45, n. 1, p. 89-98, 2010a.

LIMA, J. S. S.; SOUZA, G. S.; SILVA, S. A. Amostragem e variabilidade espacial de atributos químicos do solo em área de vegetação natural em regeneração. Revista Árvore, Viçosa, v. 34, n. 1, p. 127-136, 2010 b.

MELO-FILHO, J. F.; OLIVEIRA, A. S.; LOPES, L. C.; VELLAME, L. M. Análise estatística exploratória e variabilidade da densidade do solo em um perfil de Latossolo Amarelo coeso dos tabuleiros costeiros da Bahia. Ciência Agrotecnologica, Lavras, v. 30, n. 2, p. 199-205, 2006.

MOURA, E. G.; SERPA, S. S.; SANTOS, J. G. D.; COSTA-SOBRINHO, J. R. S.; AGUIAR, A. C. F. Nutrient use efficiency in alley cropping systems in the Amazonian periphery. Plant and Soil, The Hague, v. 335, n. 1-2, p. 363-371, 2010.
ORTIZ, J. L. Emprego do geoprocessamento no estudo da relação entre potencial produtivo de um povoamento de eucalipto e atributos do solo e do relevo. 2003. Dissertação (Mestrado em Recursos Florestais) - Escola Superior de Agricultura Luiz de Queiroz, Piracicaba.

PEREIRA NETO, O. C.; GUIMARÃES, M. F.; RALISCH, R.; FONSECA, I. C. B. Análise do tempo de consolidação do sistema de plantio direto. Revista Brasileira de Engenharia Agrícola e Ambiental, Campina Grande, v. 11, n. 5, p. 489-496, 2007.

RALISCH, R.; MIRANDA, T. M.; OKUMURA, R. S.; BARBOSA, G. M. C.; GUIMARÃES, M. F.; SCOPEL, E.; BALBINO, L. C. Resistência à penetração de Latossolo Vermelho Amarelo do Cerrado sob diferentes sistemas de manejo. Revista Brasileira Engenharia Agrícola Ambiental, Campina Grande, v. 12, n. 4, p. 381 384, 2008.

RIBEIRO, A. C.; GUIMARÃES, P. T. G.; ALVAREZ, V. V. H. Recomendação para o uso de corretivos e fertilizantes em Minas Gerais: $5^{\text {a }}$ aproximação. Viçosa: Comissão de Fertilidade do Solo do Estado de Minas Gerais, 1999. 359 p.

RICHART, A.; TAVARES FILHO, J.; BRITO, O. R.; LLANILLO, R. F.; FERREIRA, F. Compactação do solo: causas e efeitos. Semina: Ciências Agrárias, Londrina, v. 26, n. 3 , p. 321-344, 2005.

RICHTER, D. D.; BABBAR, L. I. Soil diversity in the tropics. Advision Ecology Research, v. 21, p. 315-389, 1991.

ROBERTSON, G. P. GS+: geostatistics for the environmental sciences - GS+ user's guide. Plainwell: Gamma Design Software, 1998. 152 p.

SCHAFFRATH, V. R.; TORMENA, C. A.; FIDALSKI, J.; GONCALVES, A. C. A. Variabilidade e correlação espacial de propriedades físicas de solo sob plantio direto e preparo convencional. Revista Brasileira de Ciência do Solo, Viçosa, v. 32, n. 4, p. 1369-1377, 2008.

SILVA, F. A. S.; AZEVEDO, C. A. V. Versão do programa computacional Assistat para o sistema Windows. Revista Brasileira de Produtos Agroindustriais, Campina Grande, v. 4, n. 1, p. 71-78, 2008.

SILVA, V. R.; REICHERT, J. M.; STORCK, L.; FEIJO, $\mathrm{S}$. Variabilidade espacial das características químicas do solo e produtividade de milho em um Argissolo Vermelho-Amarelo Distrófico arênico. Revista Brasileira de Ciência do Solo, Viçosa, v. 27, n. 6, p. 1013-1020, 2003. 
SILVA-NETO, S. P.; SANTOS, A. C.; LEITE, R. L. L.; DIM, V. P.; CRUZ, R. S.; PEDRICO, A.; NEVES-NETO, D. N. Análise espacial dos parâmetros da fertilidade do solo em região de ecótolo sob diferentes usos e manejos. Semina: Ciências Agrárias, Londrina, v. 32, n. 2, p. 541-552, 2011.

SOUZA, Z. M.; MARQUES-JÚNIOR, J.; PEREIRA, G. T. Variabilidade espacial de atributos físicos do solo em diferentes formas do relevo sob cultivo de cana-deaçúcar. Revista Brasileira de Ciência do Solo, Viçosa, v. 28, n. 6, p. 953-964, 2004.

SOUZA, Z. M.; MARQUES-JÚNIOR, J.; PEREIRA, G. T.; MONTANARI, R.; CAMPOS, M. C. C. Amostragem de solo para determinação de atributos químicos e físicos em área com variação nas formas do relevo. Revista Cientifica, Jaboticabal, v. 34, n. 2, p. 249-256, 2006.

STOLF, R.; FERNANDES, J.; FURLANI-NETO, V. L. Recomendações para o uso do penetrômetro de impacto, modelo IAA/Planalsucar-Stolf. São Paulo: MIC/IAA/ PNMCA-Planalsucar, 1983. 8 p.
TORMENA, C. A.; BARBOSA, M. C.; COSTA, A. C. S. Densidade, porosidade e resistência a penetração em Latossolo cultivado sob diferentes sistemas de preparo do solo. Sciencia Agrícola, Piracicaba, v. 59, n. 4, p. 795801, 2002.

TORMENA, C. A.; ROLOFF, G.; SÁ, J. C. M. Propriedades físicas do solo sob plantio direto influenciadas por calagem, preparo inicial e tráfego. Revista Brasileira de Ciência do Solo, Viçosa, v. 22, n. 2, p. 301-309, 1998.

WARRICK, A. W.; NIELSEN, D. R. Spatial variability of soil physical properties in the field. In: HILLEL, D. Applications of soil physics. New York: Academic, 1980. p. 319-344.

WEBSTER, R.; BECKETT, P. H. T. Quality and usefulness of soil maps. Nature, London, v. 219, n. 5155, p. 680-682, 1968.

WEIRICH-NETO, P. H.; ROSA, A. L. T.; GOMES, J. A. Susceptibilidade de dois solos à compactação. Revista Brasileira Engenharia Agrícola Ambiental, Campina Grande, v. 6, n. 2, p. 349-353, 2002. 
